\title{
ULTRASTRUCTURAL STUDY OF A CORNEAL KELOID
}

\author{
NADER M. SHOUKREY ${ }^{1}$ and KHALID F. TABBARA ${ }^{2}$ \\ Riyadh, Saudi Arabia
}

\begin{abstract}
SUMMARY
A 69-year-old man developed keloid overgrowth on his left cornea in response to an injury from a fingernail. The lesion was removed by superficial lamellar keratectomy and was studied by electron microscopy, and light microscopy after immunoperoxidase staining for actin. The surgical specimen revealed disorganised, anteriorly atrophied and posteriorly vascularised connective tissue stroma. The epithelium was oedematous, thin, non-keratinised, and contained cells with features of myoblastic differentiation. Stromal fibroblasts were found in several distinct ultrastructural forms including young active fibroblasts, myofibroblasts, inactive fibroblasts (fibrocytes) and fibroblasts with prominently fibrillar cytoplasm. Fibroblasts with glycogen storage and/or pseudonuclear inclusions were also seen. Macrophages and lymphocytes were scattered in the stroma, and intact nerves were also present. An irregular 2-65 $\mu \mathrm{m}$ band of $10 \mathrm{~nm}$ filament meshwork existed at the posterior border of the keloid stroma, and deep localised patches of climatic degeneration were detected.
\end{abstract}

Keloids are hypertrophic scars produced by prolonged and excessive deposition of collagen and extend beyond the confines of the original causative lesion in susceptible individuals. ${ }^{1,2}$ They usually follow trauma, ${ }^{3}$ often recur and grow larger after each excision, and can be mistaken for neoplasm. ${ }^{4,5}$ Histogenesis of keloids has been studied extensively in the skin. ${ }^{1-3}$ The developmental sequence proceeds from an early inflammatory stage to a fibroblastic stage characterised by increased vascularity, hyperplasia of juvenile fibroblasts, and production of many collagen fibres and bundles. A later fibrous stage shows increased production of collagen in tightly packed nodules and whorls, and reduction in both vascularity and juvenile fibroblasts. The compact collagen stroma then undergoes fusion and homogenisation to the final hyaline stage. The clinical course of keloid formation comprises

From: ${ }^{1}$ Research Centre, College of Applied Medical Sciences, and ${ }^{2}$ Department of Ophthalmology, College of Medicine, King Saud University, Riyadh, Saudi Arabia.

Correspondence to: Nader M. Shoukrey, PhD, College of Applied Medical Sciences, P.O. Box 10219, Riyadh 11433, Saudi Arabia. an active growth phase which persists from 6 to 12 months, leading into an inactive mature phase which may last years. ${ }^{5}$ Active spread of scarlet to pink fibrotic tissue over the wound surface corresponds histopathologically with the fibroblastic stage. Formation of the white, rigid, hypertrophic scar marks the inactive mature phase and corresponds to the fibrous and hyaline stages.

Corneal keloids are quite uncommon. Sixty-eight cases have been reported in the last five decades. ${ }^{6-18}$ Most cases followed corneal perforation or injury, sometimes with iris incarceration. Two cases have been associated with rubeola, ${ }^{9} 6$ cases with Lowe syndrome, ${ }^{13,15}$ and 5 cases existed at birth without any evidence of prior corneal injury. ${ }^{6,10,11,17,18}$ Only in 4 of these 68 cases of corneal keloids was there any ultrastructural investigation. ${ }^{15-18}$ In this study, we performed a detailed ultrastructural analysis of a post-traumatic corneal keloid. We were able to find immunohistochemical and ultrastructural evidence for the presence of 'fully developed' myofibroblasts and epithelial cells with features of myoblastic differentiation in a corneal keloid.

\section{CASE REPORT}

A 69-year-old man presented with a 5-month history of progressive painless decrease in vision in his left eye following trauma from a fingernail. He had noticed progressive opacification of his left cornea. His past ocular and medical history were unremarkable. A corrected visual acuity of 20/200 was found in the right eye, the decrease in vision being accounted for by climatic droplet keratopathy and an immature cataract. With the left eye he could see hand movements only, and slit lamp examination revealed a whitish, well-demarcated, slightly raised mass with a smooth and glistening surface covering a large area of the cornea. The unaffected sections of cornea were clear, and the anterior chamber appeared to be of normal depth, but the lens showed early nuclear sclerosis. The rest of the examination was unremarkable.

The patient was seen again 11 months later. By this time the lesion on his left cornea appeared slightly larger. It was removed completely by a superficial lamellar keratectomy. At surgery, the lesion was found to separate easily 

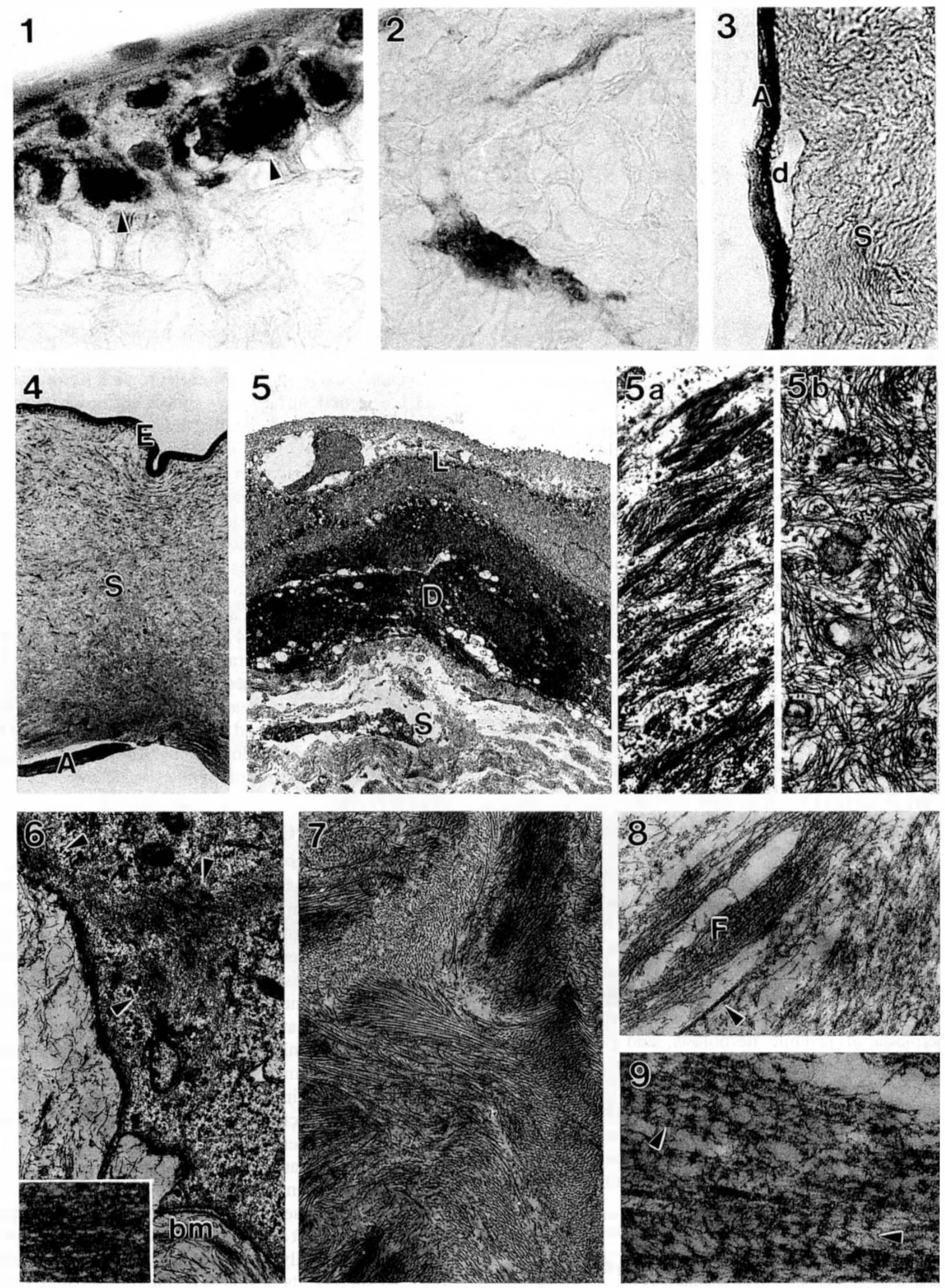
from the remaining cornea. No evidence of any previous corneal perforation could be seen biomicroscopically.

Four months after surgery the cornea remained mildly oedematous, and mild superficial scarring was observed. Eighteen months later the patient could see hand movements only and there was recurrence of the corneal keloid.

\section{HISTOPATHOLOGY}

The extirpated tissue formed an unevenly circular button, greyish-white in colour and densely opaque. It measured about $8 \mathrm{~mm}$ in diameter and $2.5 \mathrm{~mm}$ in thickness at its centre. The specimen was fixed in neutral buffered formalin, then bisected; one half was processed for light microscopy and the other for transmission electron microscopy. ${ }^{19}$ Stains for light microscopy included haematoxylin and eosin for general morphology, Alcian blue for mucopolysaccharides, PAS for glycogen, Verhoeff-Van Gieson's for climatic degeneration, and both Congo red and crystal violet for amyloid. An immunoperoxidase stain was applied by a peroxidase-antiperoxidase technique with anti-actin antibodies. ${ }^{20}$

Figs. 1-9: Opposite.

Fig. 1. Photomicrograph of keloid epithelium immunolabelled with anti-actin antibodies and stained by the peroxidaseantiperoxidase technique. Note the positive immunostaining of two basal epithelial cells (arrowheads) $(\times 640)$.

Fig. 2. Photomicrograph of keloid stroma immunolabelled with anti-actin antibodies and stained by the peroxidase-antiperoxidase technique. Note that one of two fibroblasts in the section is positively immunostained $(\times 640)$.

Fig. 3. Photomicrograph of posterior boundary of keloid stroma $(S)$ stained with the crystal violet technique, showing a limiting band, about $4 \mu \mathrm{m}$ thick, of positively staining amyloid (A). Note the discontinuous merging between stroma and amyloid-like material at $(d)(\times 800)$.

Fig. 4. Photomicrograph of transverse section of Congo-red stained keloid, showing epithelium $(E)$, stroma $(S)$ and a discontinuous, loosely attached limiting band, about $55 \mu \mathrm{m}$ thick, of positively staining amyloid-like material $(A)(\times 32)$.

Fig. 5. Electron micrograph of keloid epithelium and underlying stroma. Note basal dark cells $(D)$, superficial light cells (L), widened intercellular space and intracytoplasmic vacuolation in the epithelium, and prominent interstitial space in the underlying stroma $(S)(\times 3400)$. (a) and $(b)$ Higher magnifications demonstrating the compact and diffuse arrangements of tonofilaments in dark and light epithelial cells respectively ( $\times 49000)$.

Fig. 6. Electron micrograph of keloid basal epithelial cell showing peripheral bundles of $4 \mathrm{~nm}$ microfilaments with focal densities (arrowheads) indicating myoblastic differentiation. Note that the basement membrane $(\mathrm{bm})$ is fragmentary and thin $(\times 13$ 300). Inset: Higher magnification demonstrating the microfilaments $(\times 82$ 000).

Fig. 7. Electron micrograph of posterior part of keloid stroma showing the random and loose arrangement of collagen fibrils $(\times 7750)$.

Fig. 8. Electron micrograph of anterior part of keloid stroma showing interstitial fibrillary material $(F)$ alongside amorphous granular substance. Note the single collagen fibril (arrowhead) ( $\times 29000)$.

Fig. 9. Electron micrograph of anterior part of keloid stroma showing interstitial substance with banded appearance (arrowheads) $(\times 60$ 000).

\section{Light Microscopy}

The mass consisted of fibrovascular tissue covered with four or five layers of non-keratinised squamous epithelium which was absent in few places. Both stroma and epithelium were oedematous; Bowman's layer was entirely absent, but a fragmentary epithelial basement membrane was present.

Collagen fibres and lamellae varied in size, and coursed irregularly in the stroma of the keloid mass, with ample interstitial space anteriorly. No nodular or whorl-like patterns of collagen arrangement were detected. The interlamellar ground substance showed moderately positive reactions for mucopolysaccharides, particularly in the anterior parts of the stroma. Fibroblasts, more numerous anteriorly, exhibited moderate cytoplasmic basophilia and hyperchromatic, oblong nuclei, sometimes with an irregularly twisted appearance. Occasionally they displayed strong PAS-positive reactions, mostly anteriorly. No mitotic figures were seen. Immunohistochemical staining revealed positive reactions of some basal epithelial cells and many fibroblasts with anti-actin antibodies (Figs. 1 and 2). Actin-containing fibroblasts were present mostly in the posterior parts of the stroma. In this, also, small blood vessels and capillaries were irregularly distributed. Neither mast cells nor plasma cells were detected, but a few lymphocytes and polymorphonuclear leucocytes were present. Small punctate deposits that reacted positively with Verhoeff-Van Giesen's elastic stain, characteristic of climatic degeneration, were occasionally seen in the deepest portions of the excised tissue.

The posterior boundary of the keloid was lined by a discontinuous layer of amorphous deposits, $2-65 \mu \mathrm{m}$ in thickness. These deposits stained positively with the special amyloid stains of crystal violet and Congo red (Figs. 3 and 4). However, they did not show any birefringence with polarised light. These peripheral amorphous deposits occasionally invaded the posterior stroma of the keloid mass in irregular localised patches.

\section{Electron Microscopy}

The epithelium consisted mostly of spindle-shaped cells. In certain localised regions, epithelial cells were more electron dense ('dark') at the base than at the surface. Intracytoplasmic tonofilaments were much more numerous in the 'dark' basal cells, where they were grouped into bundles, than in the 'light', more superficial cells, where they were distributed diffusely (Fig. 5). Features of myoblastic differentiation comprising peripheral bundles of $4 \mathrm{~nm}$ microfilaments with focal densities, were detected sporadically among basal cells of the epithelium (Fig. 6). Many epithelial cells were moderately vacuolated, and intercellular spaces were frequently widened. Hemi-desmosomal junctions between basal epithelial cells and the underlying basement membrane were mostly absent. Nerves were not detected anywhere in the epithelium.

The stroma of the excised keloid mass lacked the regular orientation and dense packing of collagen lamellae peculiar to corneal stroma. Instead, these lamellae were 

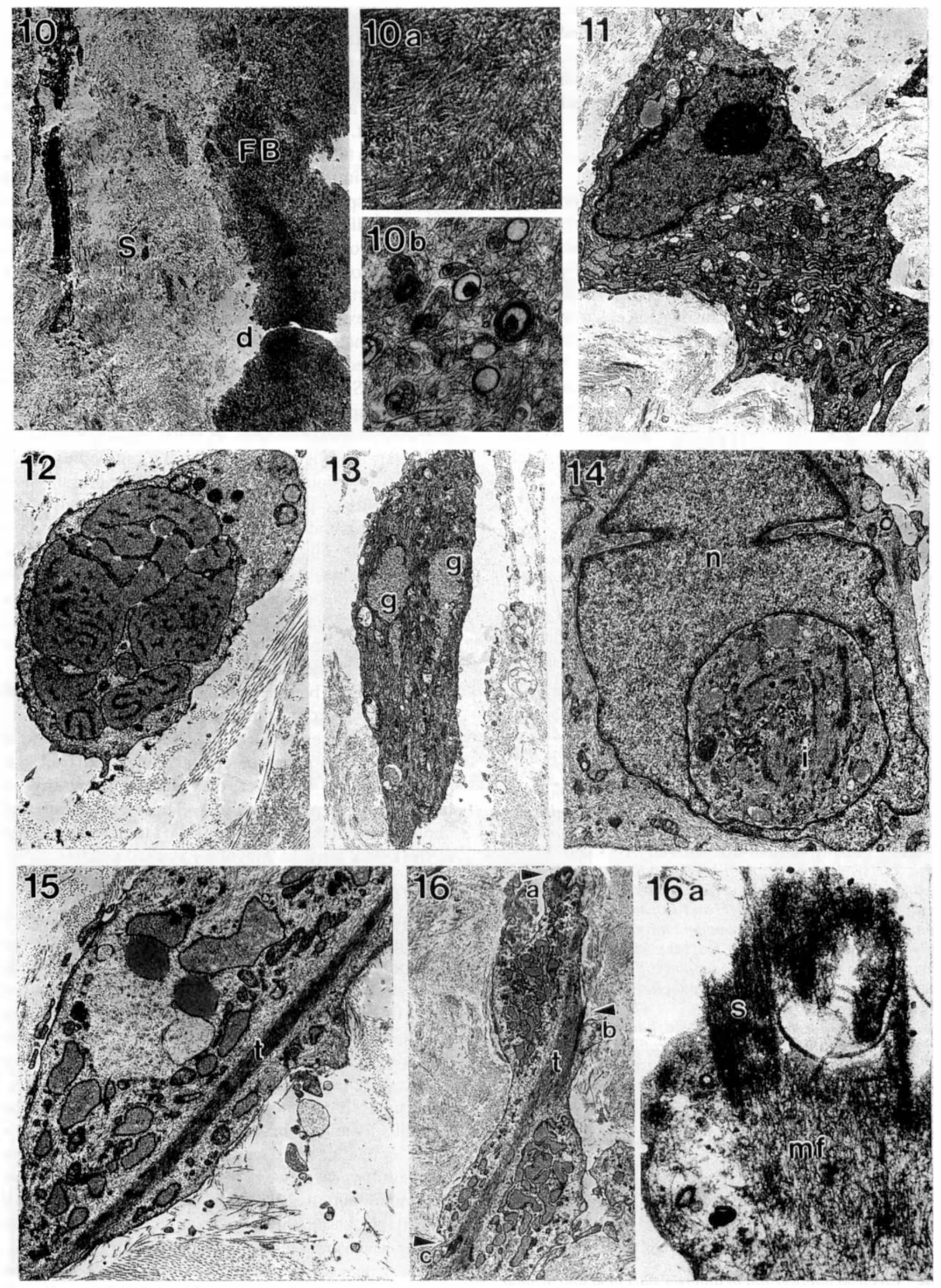
oriented haphazardly and relatively loosely (Fig. 7). Collagen fibrils varied in thickness (diameters between 15 and $37 \mathrm{~nm}$ ) and showed a distinct periodicity of $500 \mathrm{~nm}$. The interstitial spaces, most prominent in the anterior portion, were occupied partly by irregular patches of fibrillary and homogeneous material. The fibrillary material consisted of scattered bundles of thin filaments, less than $12 \mathrm{~nm}$ in diameter (Fig. 8). The homogenous material displayed, in some places, a banded appearance reminiscent of fibrous long-spacing collagen with an average periodicity of $75 \mathrm{~nm}$ (Fig. 9).

The amorphous deposits lining the posterior boundary of the keloid stroma comprised an intricate meshwork of thin filaments measuring 8-12 $\mathrm{nm}$ in diameter (Fig. 10). Towards the interface with the stroma of the keloid, this filament meshwork merged discontinuously with collagen fibrils. Numerous minute vacuoles were enmeshed singly and in clusters in this irregular limiting filament band.

The cell population of the keloid stroma consisted predominantly of fibroblasts, with moderate numbers of macrophages and a few lymphocytes. The fibroblasts were not compressed among the loosely packed collagen lamellae and tended to be oval or moderately elongated; occasionally they were stellate in shape, but they did not have any of the long cytoplasmic extensions typical of corneal keratocytes. Ultrastructurally, fibroblasts were of several distinct morphological forms. The active young variety (Fig. 11) had a well-developed rough endoplasmic reticulum, several Golgi complexes, numerous mitochondria and one or two hypertrophied marginal nucleoli, all

\section{Figs. 10-16: Opposite.}

Fig. 10. Electron micrograph of posterior part of keloid stroma showing the limiting $10 \mathrm{~nm}$ filament band $(\mathrm{FB})$. Note the discontinuous merging between keloid stroma $(S)$ and filament band at $(d)(\times 2300)$. (a) and (b) Higher magnifications of the filament band demonstrating, respectively, the random arrangement of the $10 \mathrm{~nm}$ filaments $(\times 49000)$ and the presence of vacuolated structures $(\times 23000)$.

Fig. 11. Electron micrograph of young fibroblast in keloid stroma showing well-developed rough endoplasmic reticulum, numerous mitochondria, Golgi complexes and marginal nucleolus $(\times 5000)$.

Fig. 12. Electron micrograph of fibrocyte in keloid stroma showing markedly dilated rough endoplasmic reticulum containing homogeneous material of medium density and irregular electron-dense deposits $(\times 7750)$.

Fig. 13. Electron micrograph of fibroblast in keloid stroma showing glycogen storage $(g)(\times 6000)$.

Fig. 14. Electron micrograph of fibroblast in keloid stroma showing nucleus $(n)$ with large pseudo-inclusion $(i)$. Note the presence of bundles of microfilaments with focal densities in the inclusion $(\times 10500)$.

Fig. 15. Electron micrograph of myofibroblast in keloid stroma showing the subplasmalemmal tract of microfilaments with focal densities $(t)(\times 7750)$.

Fig. 16. Electron micrograph of myofibroblast in keloid stroma most probably in contracted condition. Note the twisted subplasmalemmal tract of microfilaments $(t)$ and the three myofibroblast-stroma attachment sites $(a, b, c)(\times 3900)$. (a) A higher magnification of attachment site (a) showing an intracytoplasmic short bundle of microfilaments $(\mathrm{mf})$ perpendicular to the plasma membrane, and a more electron-dense stouter bundle $(s)$ that inserts into the stroma $(\times 49$ 000). indicative of active protein synthesis. Conversely, the relatively inactive fibrocytes contained few profiles of rough endoplasmic reticulum and a paucity of other organelles. In some fibroblasts, the rough endoplasmic reticulum was markedly dilated and contained homogeneous material of medium density, often including electron-dense deposits (Fig. 12). Some fibroblasts showed large accumulations of monoparticulate glycogen (Fig. 13), while others contained many lipid inclusions. The latter inclusions were not membrane-bound and had localised clusters of ribosomes along their periphery. Nuclei in some fibroblasts displayed pseudo-inclusions (Fig. 14) containing cytoplasmic material separated from the nuclear matrix by a double membrane, apparently an extension of the nuclear envelope. Fibroblasts with dilated rough endoplasmic reticulum, glycogen storage and nuclear pseudoinclusions were detected mostly in the anterior stroma of the keloid.

Many fibroblasts, particularly in the posterior half of the keloid stroma, had the ultrastructural characteristics of myofibroblasts. These cells incorporated subplasmalemmal tracts of microfilaments less than $4 \mathrm{~nm}$ in diameter, oriented parallel to the long axis of the cell, with focal densities along their length (Figs. 15 and 16). Intracellular, closely packed bundles of shorter microfilaments extended across the cell membrane into the extracellular space at opposite ends of the long axis of the cell (Fig. 16). Furthermore, occasional nuclei were convoluted, with an accordion-like appearance. However, desmosomes joining myofibroblasts to adjacent cells were not detected.

The varieties of fibroblasts and fibrocytes described above all had fibrillar cytoplasm. The degree of fibrillation ranged from sparse to dense (Figs. 17 and 18), but the sparsely fibrillated cells were more numerous than the densely fibrillated ones.

Macrophages were present mostly in the anterior keloid stroma. Some of these cells had many phagocytic vacuoles containing collagen fibrils (Fig. 19); others displayed prominent cytoplasmic processes but with only occasional lysosomes and phagocytic vacuoles present. Polymorphonuclear leucocytes were only seen within the lumina of blood vessels. These vessels had round to oval lumina that were delineated by endothelium and related pericytes. A thickened lamellar basal lamina was present in most blood vessels observed (Fig. 20). The innermost lamella was intimately applied to the endothelium and embraced the pericytes.

Nerves were sporadically seen in the posterior portion of the keloid mass. They consisted of numerous nonmyelinated axons, enclosed in Schwann cells (Figs. 21 and 22). Highly electron-dense, irregularly shaped bodies, limited by a single membrane were seen in some nerves. These may well represent lipofuscin-containing residual bodies, the presence of which is related to our patient's advanced age.

The deposits relating to climatic degeneration which were detected in the posterior stroma of the keloid mass consisted of highly electron-dense homogeneous globules of variable size, embedded in a vacuolated matrix of 

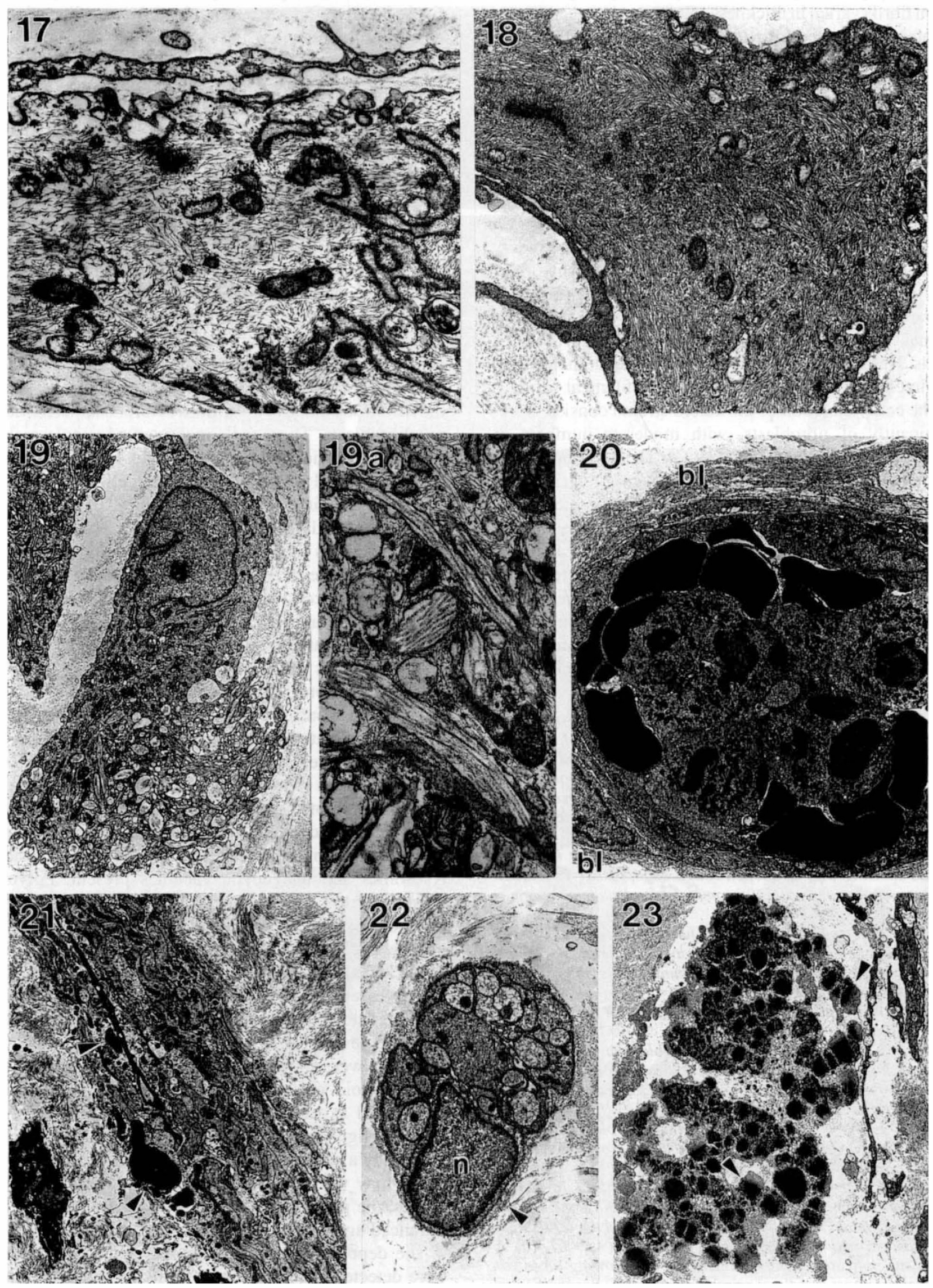
granular amorphous material (Fig. 23). These electrondense globules merged with the matricial amorphous material and in some places displayed a biphasic appearance.

\section{DISCUSSION}

O'Grady and Kirk ${ }^{10}$ believe that the clinical appearance of hypertrophic scars is specific enough to justify their inclusion in the differential diagnosis of any glistening, white, protuberant corneal mass; biopsy will define the final diagnosis. In our patient, the diagnosis of the corneal lesion as keloid was based on five clinical features:

1. The lesion was greyish-white, elevated, firm, smooth, shiny and well demarcated from adjacent tissue.

2. There was a clinical history of an injury from a fingernail preceding development of the lesion.

3. There was continued growth of the lesion for over 18 months.

4. Corneal thickening was caused by fibrovascular hyperplasia, thought to be secondary to scar formation even though no microscopic evidence of any trauma could be detected.

5. The lesion, devoid of any displaced dermal elements, covered a large area of the corneal surface, well beyond the presumed localised site of the presumed causative fingernail injury.

These findings, while not precluding corneal dermoid and primary fibroma, ${ }^{6}$ are most consistent with the diagnosis of keloid. In contrast to some earlier case reports, ${ }^{6-9,11,16}$ in our patient there was no evidence of corneal perforation, or of iris or lens incarceration. This suggests that the keloid in our case originated from primary corneal reparative processes rather than from a direct participation of the iris or lens in scar formation. ${ }^{8}$

Histopathologically, we found features of our keloid specimen different from those of corneal keloids reported

Figs. 17-23: Opposite.

Fig. 17, 18. Elecron micrographs of two fibrocytes in the keloid stroma showing sparsely and densely fibrillar cytoplasm respectively $(\times 18000)$.

Fig. 19. Electron micrograph of macrophage in keloid stroma showing numerous phagocytic vacuoles, some of which enclose collagen fibrils $(\times 3900)$. (a) A higher magnification of the phagocytic vacuoles with collagen fibrils $(\times 18$ 000).

Fig. 20. Electron micrograph of small blood vessel in keloid stroma showing thickened basal lamina (bl) and intraluminal polymorphonuclear leucocytes $(\times 3250)$.

Fig. 21. Electron micrograph of longitudinal section of a nerve in keloid stroma showing numerous unmyelinated axons and highly electron-dense bodies (arrowheads) probably representing lipofuscin residual bodies $(\times 5000)$.

Fig. 22. Electron micrograph of transverse section of another nerve in keloid stroma showing many unmyelinated axons, a Schwann cell with prominent nucleus (n) and basal lamina (arrowhead) $(\times 5000)$.

Fig. 23. Electron micrograph of an area of climatic degeneration in the most posterior part of the keloid stroma showing the biphasic nature of many climatic degeneration globules (arrowheads) as they merge with amorphous granular vacuolated matrix $(\times 10000)$. in the literature. The epithelial coat of our keloid, a continuation of the corneal epithelium, was non-keratinised, thin and denervated. Apparently as a result of a degenerate basement membrane, it had detached completely at some loci along the keloid surface. The epithelium was oedematous and displayed the dark-cell/light-cell phenomenon possibly attributable to excessive dehydration. ${ }^{21}$ In comparison, epithelia of corneal keloids in cases in the literature have been reported as keratinised and hyperplastic, ${ }^{6-10,15-17}$ occasionally with mucoid degeneration $^{6}$ and lipid infiltration. ${ }^{17}$

The detection of epithelial cells with features of myoblastic differentiation, in our case by immunological and ultrastructural techniques, has no parallel in the literature on keloids. Such epithelial cells have been reported, however, in healing experimental skin wounds, ${ }^{22}$ and in regenerating epidermal cells following experimental production of blisters. ${ }^{23}$ Under these conditions, the development of contractile elements in epidermal cells was shown to be reversible. Such contractile elements were thought to enable individual cells to move, in order to rearrange themselves in response to specific changes in their microenvironment. How this proposition applies to epithelial cells of a keloid is not clear. Further studies are needed to investigate the manner in which contractile elements develop in the cells of an epithelium overlying a keloid.

Vascularisation of corneal keloids was described in earlier reports as diffuse, ${ }^{6,8,10-14,17,18}$ anterior, ${ }^{9}$ or as diffuse but with more vessels located anteriorly. ${ }^{6,715}$ In our case, small blood vessels were detected only in the posterior portions of the keloid stroma, thus presumably posing circulatory compromise of the anterior stroma, conceivably promoting oedema. This hypothesis is consistent with our observation that most of the blood vessels had thickened basal laminae, a feature which is thought by Vracko and Benditt ${ }^{24}$ to reflect a high turnover of the vascular endothelium, leading to a degenerative event and enhancing oedema.

The avascular, oedematous anterior keloid stroma was atrophic, with ample interstitial space that was incompletely occupied by amorphous and fibrillary material. The 'unoccupied' portions might represent either oedematous swelling or degenerative foci where disintegration of collagen fibrils occurred and left irregular spaces. ${ }^{25}$ Furthermore, the ingress of water, accelerated by existing oedema, might explain the marked dilatation of the rough endoplasmic reticulum seen in some fibroblasts in the anterior stroma. It might also have caused an increase in cytoplasmic volume, leading to evagination of cytoplasmic material into the nucleus and thus accounting for nuclear pseudo-inclusions. ${ }^{26}$ On the other hand, accumulation of glycogen in stromal fibroblasts may be interpreted in terms of the level of fibroblast activity: it may indicate diminished usage in the relatively inactive fibrocytes, or it may express increased metabolic activity in young active fibroblasts.

In the stroma of our keloid specimen, whorl and nodule 
formations of collagen, characteristic of dermal keloids, were not found. Cibis et al. ${ }^{15}$ confirming our finding, ascribed this inconsistency to the peculiar architecture of corneal collagen. However, the reshaping of corneal collagen in our specimen may be correlated with the observed macrophages containing phagocytosed collagen. Their presence might signify reabsorption and remodelling of collagen, characteristic of healing wounds in $\operatorname{man}^{27}$ and guinea pigs. ${ }^{28}$

Easy separation of the keloid mass from the remaining cornea at the time of surgery implied a loose attachment between the two tissues. Microscopically, this correlated with the zone of $10 \mathrm{~nm}$ filaments deposited along the keloid-cornea interface. These deposits may represent either collagen precursors, products of collagen degeneration, amyloid fibrils, or some other substance. When viewed by electron microscopy, the $10 \mathrm{~nm}$ filaments were concentrated in a limiting band far removed from fibroblasts and loosely attached to the keloid stroma, making it less likely - but still a viable possibility - that they consisted of immature forms of collagen. ${ }^{29}$ Histochemically, these filaments reacted positively with special stains for amyloid (Congo red and crystal violet) but failed to exhibit birefringence with polarised light. If such failure is attributed to the disorderly arrangement of these filaments in their dense deposits, ${ }^{30}$ the possibility that they are amyloid is not precluded. The considerable variation in thickness $(2-65 \mu \mathrm{m})$ of this limiting band of filaments could possibly be attributed to an unevenness in the plane of splitting when the keloid mass was separated from the cornea during surgery.

The presence of climatic degeneration at the base of our keloid specimen is an incidental finding that was not unexpected. The clusters of climatic degeneration globules may have originated in the underlying corneal stroma and are consistent with the patient's age .

Two important findings in our case are the detection in the keloid stroma of both fully developed myofibroblasts and densely fibrillated fibroblasts. James et $a l .^{31}$ believe that the active phase of keloid growth is correlated with a predominance of myofibroblasts, and this is consistent with Chiu and McFarlane's observations in Dupuytren's contracture. ${ }^{32}$ It follows that the presence of myofibroblasts, in moderate numbers relative to the total fibroblast population, in the corneal keloid of our patient may suggest that it was nearing its inactive, mature phase. This is further supported by:

1. The presence of moderate amounts of mucopolysaccharides only anteriorly. ${ }^{3}$

2. The absence of desmosomes joining myofibroblasts to adjacent cells. $^{33}$

3. The detection of moderate numbers of densely fibrillated fibroblasts.

Rudolph et al..$^{33}$ observed a similar type of densely fibrillated fibroblast in the late, contracted phase of a granulated wound in a pig. These authors believed that the densely packed filaments were responsible for keeping the fibro- blasts in a contracted state following the disappearance of the myofibroblasts, allowing time for reorganisation of collagen into a fixed rigid scar. It is noteworthy that three of the four previous ultrastructural studies on corneal keloids $^{15-17}$ did not report any myofibroblasts. Possible explanations might be either that those cases, being mature inactive keloids, reflected the end stage in which myofibroblasts are absent or inactive, or merely that myofibroblasts were overlooked. The documentation by Holbach et al. ${ }^{18}$ of myofibroblasts in corneal keloids of children was based on the ultrastructural presence of long bundles of 5-6 nm actin-like microfilaments with scattered fusiform densities. However, these authors did not report other equally specific ultrastructural features as outlined by Gabbiani et al.$^{34}$ regarding cell membrane attachment sites, cell surface differentiation and the accordion-like morphology of the nucleus resulting from cell contraction. It may be that the myofibroblasts reported by Holbach $e t$ al. were merely fibroblast-myofibroblast transition forms.

The presence of large intact nerves in the keloid stroma of our specimen was an unexpected finding. Peripheral nerves invariably lose their sheath of Schwann as they extend into the central cornea and terminate as bare axons in the stroma and around keratocytes. ${ }^{35}$ Dyer and Enna ${ }^{36}$ suggest that intact nerves are essential for the development of normal and hypertrophic scars. This is based on their observation that the development of dermal keloids is rare in patients with denervated skin, as in lepromatous leprosy.

It is obvious that the role of each of the pathological processes involved in the formation of a corneal keloid (innervation, fibroblastic transformations, vascularisation, etc.) is speculative. The development of experimental models of corneal keloid formation would be essential for a better understanding of these processes.

Key words: Amyloid, Cornea, Epithelial cells with myoblastic differentiation, Keloid, Myofibroblasts, Ultrastructure.

\section{REFERENCES}

1. Lever WF, Schaumburg-Lever G. Tumors of fibrous tissue. In: Histopathology of the skin. Philadelphia: Lippincott, 1984:604-5.

2. Linares HA, Kischer CW, Dobrkovsky M, Larsen DL. The histiotypic organisation of the hypertrophic scar in humans. J Invest Dermatol 1972;59:323-31.

3. Mancini RE, Quaife JV. Histogenesis of experimentally produced keloids. J Invest Dermatol 1962;38:143-81.

4. Cohen K, Diegelmann RF, Keiser HR. Collagen metabolism in keloid and hypertrophic scars. In: Longaere JJ, editor. The ultrastructure of collagen. Illinois: CC Thomas, 1976:199.

5. Konuralp HZ. Keloids and hypertrophic scars: their properties, principles and therapy. In: Longaere JJ, editor. The ultrastructure of collagen. Illinois: CC Thomas, 1976: 390-448.

6. Smith HC. Keloid of the cornea. Trans Am Ophthalmol Soc 1940;38:519-38.

7. Fenton RH, Tredici TJ. Hypertrophic comeal scars (keloids). Surv Ophthalmol 1964;9:561-6.

8. Farkas TG, Znajda JP. Keloid of the cornea. Am J Ophthalmol 1968;66:319-23.

9. Frederique G, Howard RO, Bonuik V. Corneal ulcers in rubeola. Am J Ophthalmol 1969;68:996-1003. 
10. O'Grady RB, Kirk HQ. Corneal keloids. Am J Ophthalmol 1972;73:206-13.

11. Brown SI. Corneal transplantation of the infant cornea. Trans Am Acad Ophthalmol Otolaryngol 1974;78: OP461-6.

12. Shukla IM, Arora NP, Arora MM. Corneal keloid. Ind J Ophthalmol 1975;23:18-9.

13. Cotlier E. Discussion: In: Bergsma D, Born AJ, Cotlier E, editors. The eye and inborn errors of metabolism. New York: Liss, 1976:579-95.

14. Pouliquen Y, Dehrmy P, Chauvaud M. Une lesion cornéenne bien particulière: pannus hypertrophique. Bull Soc Ophthalmol Fr 1979;79:501-2.

15. Cibis GW, Tripathi RC, Tripathi BJ, Harris DJ. Corneal keloid in Lowe's syndrome. Arch Ophthalmol 1982;100: 1795-9.

16. Lahav M, Cadet JC, Chirambo M, Rehani U, Ishii Y. Corneal keloids: a histopathological study. Graefe's Arch Clin Exp Ophthalmol 1982;218:256-61.

17. Weiner MJ, Albert DM. Congenital corneal keloid. Acta Ophthalmol (Copenh) 1989;67:188-96.

18. Holbach LM, Font RL, Shivitz IA, Jones DB. Bilateral keloid like myofibroblastic proliferations of the cornea in children. Ophthalmology 1990;97:1188-93.

19. Zimmerman LE, Font RL, Tso MOM, Fine BS. Application of electron microscopy to histopathologic diagnosis. Trans Am Acad Ophthalmol Otolaryngol 1972;76:101-7.

20. Eishi Y, Hatakeyama S, Takemura T, Hajikano H, Hirokawa K. Demonstration of various antigens on paraffin sections of formalin fixed tissues: trypsin treated indirect peroxidaselabelled antibody technique. Bull Tokyo Med Dental Univ 1981;28:27-43.

21. Ghadially FN. Ultrastructural pathology of the cell and matrix. London: Butterworth, 1982:330-7, 704-9.

22. Krawczyk WS. A pattern of epidermal cell migration during wound healing. J Cell Biol 1971;49:247-63.

23. Gabbiani G, Ryan GB. Development of a contractile appara- tus in epithelial cells during epidermal and liver regeneration. J Submicross Cytol 1974;6:143-57.

24. Vracko R, Benditt EP. Capillary basal lamina thickening. J Cell Biol 1970;47:281-5.

25. Hidayat AA, Risco JM. Amyloidosis of corneal stroma in patients with trachoma. Ophthalmology 1989;96:1203-11.

26. Sobel HJ, Schwarz R, Marguet E. Non-viral nuclear inclusions. I. Cytoplasmic invaginations. Arch Pathol 1969;87:179-92.

27. Ross R, Odland G. Human wound repair. II. Inflammatory cells, epithelial-mesenchymal interrelations and fibrogenesis. J Cell Biol 1968;39:152-68.

28. Williams G. The late phase of wound healing: histological and ultrastructural studies of collagen and elastic tissue formation. J Pathol 1970;102:61-8.

29. Shelburne JD. Ultrastructural alterations in pathologic states. In: Garner A, Klintworth GK, editors. Pathology of ocular disease. New York: Dekker, 1982:349.

30. Yanoff M, Fine BS. Cornea. In: Ocular pathology. Philadelphia: Harper \& Row, 1982:306-82.

31. James WD, Besancency CD, Odom RB. The ultrastructure of a keloid. J Am Acad Dermatol 1980;3:50-7.

32. Chiu HF, McFarlane RM. Pathogenesis of Dupuytren's contracture: a correlative clincopathologic study. J Hand Surg 1978;3:1-10.

33. Rudolph R, Gubers S, Suzuki M, Woodward M. The life cycle of the myofibroblast. Surg Gynecol Obstet 1977;145: 389-94.

34. Gabbiani G, Ryan GB, Majno G. Presence of modified fibroblasts in granulation tissue and their possible role in wound contraction. Experientia 1971;27:549-55.

35. Hogan MJ, Alvarado JA, Weddell JE. The cornea. In: Histology of the human eye. Philadelphia: WB Saunders, 1971:62-4.

36. Dyer FR, Enna CD. Ultrastructure of keloid: an unusual incident involving lepromatous leprosy. Int $\mathrm{J}$ Dermatol $1975 ; 14: 743-54$ 